\title{
Ingesta alimentaria y evaluación antropométrica en niños escolares de Buenos Aires
}

\author{
Food intake and anthropometric evaluation in school-aged \\ children of Buenos Aires
}

\author{
Dra. Irina Kovalskys ${ }^{a}$, Lic. Paula Indart Rougiera , Lic. María Paz Amigo \\ Lic. María José De Gregorio ${ }^{b}$ Dra. Cecile Rausch Herscovicia y Lic. Marcela Karner ${ }^{a}$
}

\section{RESUMEN}

Introducción. Los principales objetivos de este estudio fueron analizar la ingesta alimentaria y determinar la prevalencia de sobrepeso y obesidad en niños escolares.

Población y métodos. Se incluyeron niños de $5^{\circ}$ grado provenientes de 80 escuelas públicas, aleatoriamente seleccionadas, del área metropolitana de Buenos Aires. Los datos de ingesta fueron recolectados a través del recordatorio de $24 \mathrm{~h}$ y de la frecuencia de consumo semanal. También se recabaron datos de peso y talla.

Resultados. La muestra quedó constituida por 1673 niños (854 mujeres y 819 varones), con una edad promedio de 10,9 años. La ingesta calórica promedio fue de $2316 \mathrm{kcal} /$ día $( \pm 888$ ) con un porcentaje de adecuación respecto de la ingesta dietética de referencia de 115,8\%. El 21,3\% de los niños evaluados presentó sobrepeso y el 14,3\% obesidad. La ingesta media de fibra fue de $9 \mathrm{~g} /$ día (ingesta dietética de referencia: $28 \mathrm{~g} /$ día, $32,1 \%$ adecuación). Solo el $2 \%$ de los niños cumpliócon la recomendación de ingesta de verduras y el $17 \%$ con la de frutas. Los alimentos de alta densidad calórica más frecuentemente consumidos fueron: gaseosas/jugos, golosinas, galletitas dulces y aderezos de alto contenido graso. Conclusiones. La elevada ingesta calórica hallada, sumada a la prevalencia de sobrepeso y obesidad en la población estudiada, justifican la necesidad de implementar programas de educación nutricional para estimular una alimentación más saludable.

Palabras clave: ingesta, niños escolares, nutrición, sobrepeso, obesidad.

http://dx.doi.org/10.5546/aap.2013.9

\section{INTRODUCCIÓN}

El valor de la nutrición en la infancia ha cobrado relevancia debido al mayor conocimiento de su influencia tanto en el óptimo crecimiento y desarrollo como en la prevención de enfermedades del adulto.

Las necesidades de macronutrientes y micronutrientes en las diferentes etapas de la vida pediátrica son de suma importancia, tanto para definir políticas y programas de salud, como para construir las bases de las ingestas dietéticas de referencia.

El aumento de la ingesta calórica es uno de los principales factores asociados con la obesidad y las enfermedades crónicas de la vida adulta. ${ }^{1,2}$ Adicionalmente, un progresivo deterioro de la calidad alimentaria, con un incremento en el porcentaje de grasas saturadas y un menor consumo de frutas y verduras, parece insinuarse como la modalidad alimentaria generalizada de la nueva era. ${ }^{3}$

El análisis de las características de la ingesta alimentaria de cada población y su asociación con la obesidad es una de las prioridades investigativas de la Organización Mundial de la Salud (OMS).

La falta de datos, a nivel poblacional, sobre ingesta alimentaria y patrones de consumo locales en niños argentinos motivó la realización de este estudio, cuyos objetivos se centraron en:

Objetivo primario

- Analizar y evaluar la ingesta alimentaria y determinar la prevalencia de sobrepeso y obesidad en niños escolares.

\section{Objetivos secundarios}

- Calcular la composición nutricional de la dieta y comparar los valores obtenidos con las ingestas dietéticas de referencia (IDR) según edad y sexo.

- Determinar la frecuencia de consumo semanal de alimentos calóricamente densos, de frutas y verduras. 


\section{POBLACIÓN Y MÉTODOS}

El presente es un estudio descriptivo de corte transversal con selección muestral aleatoria que representa a la población de niños de $5^{\circ}$ grado que asisten a escuelas públicas del área metropolitana de Buenos Aires (Ciudad Autónoma de Buenos Aires y conurbano bonaerense). La recolección de datos se realizó durante el período junio-diciembre de 2005.

Para cubrir todo el espectro socioeconómico, se eligieron sistemáticamente con arranque aleatorio y probabilidades proporcionales a su población, en el caso de la Ciudad Autónoma de Buenos Aires, 16 fracciones censales según el Censo Nacional de Población y Vivienda 1991, ${ }^{4}$ y en el conurbano bonaerense 64 localidades según el Censo Nacional de Población, Hogares y Viviendas $2001 .^{5}$ En ambos casos, dichas unidades censales fueron ordenadas de acuerdo al hacinamiento (variable proxy de nivel socioeconómico). En las fracciones y localidades mencionadas se seleccionaron aleatoriamente 80 escuelas públicas primarias a partir de los listados provistos por los organismos públicos correspondientes. Este diseño asegura una muestra representativa del área metropolitana de Buenos Aires, que reproduce proporcionalmente el perfil socioeconómico de la población bajo análisis, y puede ser procesada con un programa informático estadístico elaborado en forma específica para muestras aleatorias simples.

Se relevaron los niños de $5^{\circ}$ grado cuyos padres otorgaron el consentimiento informado por escrito para su participación en esta investigación.

Se excluyeron del análisis los niños que, al momento de la evaluación, declararon presentar alguna enfermedad aguda o crónica que comprometiera su estado nutricional. También se excluyeron los niños que declararon haber modificado sus patrones alimentarios como consecuencia de una enfermedad preexistente.

Para analizar la ingesta alimentaria se utilizaron dos instrumentos: el recordatorio de $24 \mathrm{~h}$ y la frecuencia de consumo semanal.

\section{Recordatorio de 24 horas}

El objetivo del recordatorio de $24 \mathrm{~h}$ fue ayudar al niño a recordar la totalidad de alimentos y bebidas consumidas el día anterior. Esta encuesta fue realizada por nutricionistas, quienes, para lograr rescatar y describir con certeza el total de alimentos y bebidas ingeridas en las $24 \mathrm{~h}$ previas, utilizaron modelos visuales de alimentos, ${ }^{6}$ réplicas de alimentos e instrumentos de medición caseros (plato, vaso, etc.).
Las encuestas se realizaron los días martes, miércoles y jueves para evitar incluir datos relativos a los días de fin de semana.

Los datos relevados se cargaron en una base de datos y se analizaron mediante un programa informático, especialmente diseñado sobre la base de la tabla de composición química de los alimentos CENEXA, ${ }^{7}$ que determinó el contenido de calorías y de macronutrientes. Luego, estos valores se compararon con las IDR. . $^{8,11}$

\section{Frecuencia de consumo semanal}

Se utilizó un cuestionario elaborado sobre la base de una lista de alimentos y bebidas que responden a los patrones de ingesta locales y que pueden estar asociados fuertemente con el desarrollo de sobrepeso y obesidad. En este listado se incluyeron tanto alimentos saludables (frutas y verduras) como alimentos de alta densidad calórica (hamburguesas/panchos, rebozados de pollo/pescado, choripán, pizzas/empanadas, galletitas dulces, golosinas (sin chocolate), snacks/productos de copetín, gaseosas/jugos, chocolate, tortas/facturas, frituras, manteca/ aderezos grasos).También se establecieron categorías para determinar la cantidad de veces que los niños consumían dichos alimentos y bebidas en la semana.

Para evaluar la ingesta de frutas y verduras se comparó el consumo diario con la recomendación de las Guías Alimentarias para la Población Argentina ("consumir cinco porciones entre frutas y verduras por día"). ${ }^{12}$ A partir de esta recomendación, se consideró "adecuado" el consumo de dos o más porciones de fruta diarias, "aceptable" el consumo de al menos una porción diaria $\mathrm{e}$ "inadecuado" cuando no eran consumidas diariamente. En el caso de las verduras, se consideró "adecuado" el consumo de dos o más porciones diarias (crudas/cocidas), "aceptable" el consumo de al menos una porción diaria (cruda/cocida) $\mathrm{e}$ "inadecuado" cuando no eran consumidas en forma diaria.

Ambas encuestas (recordatorio de $24 \mathrm{~h}$ y frecuencia de consumo semanal) fueron realizadas por 20 licenciados en Nutrición, especializados en antropometría, que fueron previamente capacitados en el manejo de las encuestas y de los instrumentos de evaluación antropométrica con el fin de lograr una estimación precisa de las cantidades de alimentos o bebidas y de las mediciones antropométricas. Se destinó un promedio de 40 minutos de entrevista por niño. 


\section{Antropometría}

Para medir el peso y la talla se utilizaron balanzas portátiles CAM (Buenos Aires, Argentina), con resolución de hasta 100 gramos, y estadiómetros desmontables de pared SECA Bodymeter 208 stadiometers (Hamburgo, Alemania), con resolución de hasta un milímetro. A partir de estos datos, se calculó el índice de masa corporal (IMC) y se lo comparó con los estándares OMS 2007 (sobrepeso: puntaje $Z>1 \mathrm{DE}$ y obesidad: puntaje $Z>2$ DE). ${ }^{13}$ Para realizar dicho análisis antropométrico se consideró solamente a los niños de entre 10 y 11,9 años de edad; por lo que la muestra quedó constituida por 1588 niños (817 mujeres y 771 varones).

\section{Procesamiento estadístico}

Los datos fueron procesados mediante el programa estadístico SPSS 10.0. Para el análisis de los datos antropométricos se calculó la media y la dispersión (desvío estándar) con el programa epi info versión 6.04 (1996, Centers for Disease ControlOMS, Atlanta, EE.UU.). Las comparaciones entre sexo y categoría de peso se realizaron con la prue- ba t de diferencia de proporciones. La significación estadística se estableció en $p<0,05$. Para estudiar la ingesta calórica y de macronutrientes se calculó la media, el desvío estándar (DE) y se realizó una distribución percentilar de la propia muestra; para lo cual se calcularon los puntos de corte para los percentilos 25, 50 y 75 (Pc25, Pc50 y Pc75).

\section{RESULTADOS}

La muestra quedó constituida por 1673 niños (854 mujeres y 819 varones), con una edad promedio de 10,9 años. El 95\% de los niños tenía entre 10 y 11 años y solo el 5\% tenía 12 años.

Las características de la muestra evaluada y la prevalencia de sobrepeso y obesidad según sexo se presentan en la Tabla 1. Estos resultados se expresan para el $95 \%$ de la población $(n=1588)$. No se observan diferencias significativas con los resultados de la muestra total $(n=1673)$.

La Tabla 2 muestra los valores de ingesta calórica y de composición nutricional según sexos expresados en media, DE y percentilos. Además, incluye el porcentaje de adecuación respecto de las IDR para la media de la muestra.

TABla 1. Descripción de la muestra y prevalencia de sobrepeso y obesidad para el total de la población y según sexo

\begin{tabular}{lccc}
\hline & Total $(\mathbf{n}=\mathbf{1 5 8 8})$ & Mujeres $(\mathbf{n}=\mathbf{8 1 7})$ & Varones $(\mathbf{n}=\mathbf{7 7 1})$ \\
\hline Edad (años) & $10,9( \pm 0,4)^{*}$ & $10,9( \pm 0,4)^{*}$ & $10,9( \pm 0,4)^{*}$ \\
Peso $(\mathrm{kg})$ & $39,2( \pm 9,5)^{*}$ & $39,1( \pm 9,4)^{*}$ & $39,3( \pm 9,6)^{*}$ \\
Talla $(\mathrm{cm})$ & $143,1( \pm 6,9)^{*}$ & $143,5( \pm 7,0)^{*}$ & $142,6( \pm 6,8)^{*}$ \\
IMC $\left(\mathrm{kg} / \mathrm{m}^{2}\right)$ & $19,01( \pm 3,55)^{*}$ & $18,83( \pm 3,50)^{*}$ & $19,18( \pm 3,64)^{*}$ \\
Sobrepeso $(\%)^{\mathrm{a}}$ & 21,3 & 21,1 & 21,5 \\
Obesidad $(\%)^{\mathrm{b}}$ & 14,3 & $10,6^{* *}$ & $17,9^{* *}$ \\
\hline
\end{tabular}

* Media (Desvío Estándar), ${ }^{* *}$ Diferencia significativa $\mathrm{p}<0,01$ entre sexos.

a. Puntaje Z > 1 DE. OMS 2007; b. Puntaje Z > 2 DE. OMS 2007.

TABLA 2. Datos de ingesta calórica y de composición nutricional según sexos expresados en media, DE (desvío estándar) y percentilos. Porcentaje de adecuación respecto de las IDR (en comparación con valores de la media poblacional)

\begin{tabular}{|c|c|c|c|c|c|c|c|c|c|c|c|}
\hline \multirow{3}{*}{$\begin{array}{l}\text { Energía/ } \\
\text { Nutriente }\end{array}$} & \multirow{3}{*}{ Media } & \multirow{3}{*}{$\mathrm{DE}$} & \multirow{2}{*}{\multicolumn{3}{|c|}{ Total $(n=1673)$}} & \multirow{3}{*}{ IDR $^{*}$} & \multirow{3}{*}{$\begin{array}{c}\% \\
\text { Adecuación }\end{array}$} & \multicolumn{2}{|c|}{$\begin{array}{l}\text { Mujeres } \\
(n=854)\end{array}$} & \multicolumn{2}{|c|}{$\begin{array}{l}\text { Varones } \\
(\mathrm{n}=819) \\
\end{array}$} \\
\hline & & & & & & & & Media & DE & Media & $\mathrm{DE}$ \\
\hline & & & 25 & 50 & 75 & & & & & & \\
\hline $\begin{array}{l}\text { Energía total } \\
(\mathrm{kcal} / \mathrm{d})\end{array}$ & 2316 & 888 & 1691,8 & 2175,7 & 2829,8 & 2000 & 115,8 & 2239 & 872 & 2396 & 898 \\
\hline $\begin{array}{l}\text { Hidratos de } \\
\text { Carbono }(\mathrm{g} / \mathrm{d})\end{array}$ & 307 & 120 & 223,1 & 291,4 & 368,2 & $225-325(275)$ & 111,6 & 297,5 & 118 & 316 & 121,3 \\
\hline Proteínas (g/d) & 88 & 40,1 & 59,8 & 82,2 & 111,1 & $50-75(62,5)$ & 140,8 & 84,7 & 40 & 92 & 40 \\
\hline Lípidos totales $(\mathrm{g} / \mathrm{d})$ & 84 & 44,9 & 51,1 & 76,5 & 107,1 & $55-77,7(66,35)$ & 126,6 & 81,3 & 43,2 & 86,8 & 46,5 \\
\hline Fibra (g/d) & 9 & 6,1 & 4,6 & 8 & 12,2 & 28 & 32,1 & 8,5 & 5,6 & 9,6 & 6,5 \\
\hline
\end{tabular}

* Food and Nutrition Board. Institute of Medicine. National Academy of Sciences.

IDR: Ingesta dietética de referencia. 
$\mathrm{Al}$ analizar la ingesta de macronutrientes encontramos que su distribución es armónica; para la media poblacional, los hidratos de carbono representan el 53\% de la ingesta calórica, las proteínas el $15 \%$ y las grasas el $32 \%$.

Los varones consumen 187 y $199 \mathrm{kcal}$ más que las mujeres en los percentilos 50 y 75, respectivamente. En el Pc50 las mujeres consumen $2091 \mathrm{kcal}$ y los varones $2278 \mathrm{kcal}$, y en el Pc75 las mujeres ingieren $2716 \mathrm{kcal}$ y los varones $2915 \mathrm{kcal}$. Los porcentajes de energía que provienen de los hidratos de carbono, proteínas y grasas se mantienen estables e independientes del valor calórico total.

La ingesta media de colesterol es de 295,8 $\mathrm{mg} /$ día.

En relación con la ingesta diaria de frutas y verduras según la recomendación de las Guías Alimentarias para la Población Argentina, ${ }^{12}$ hallamos que el consumo de frutas es adecuado en el $17 \%$, aceptable en el $26 \%$ e inadecuado en el $57 \%$ de la población estudiada. En cuanto a las verduras, el consumo es adecuado en solo el $2 \%$, aceptable en el $12 \%$ e inadecuado en el $86 \%$ de los niños evaluados.

En la Tabla 3 se observa la frecuencia de consumo semanal de los alimentos o los grupos de alimentos de alta densidad calórica.

\section{DISCUSIÓN}

Este estudio revela que la ingesta calórica promedio en niños de 10-12 años de edad es de 2316 $\mathrm{kcal} /$ día. El valor calórico medio hallado resulta un $15,8 \%$ superior a la recomendación. Un $25 \%$ de la población consume 2829,8 kcal o más, superando en un $40 \%$ lo recomendado (Pc75). En la población estudiada la prevalencia de sobrepeso (incluida la obesidad) es de 35,6\%. La distribución de la alimentación es armónica; es decir, conserva una proporción adecuada de hidratos de carbono, proteínas y grasas. Pero, a medida que aumenta la ingesta calórica, los valores absolutos en gramos por día superan la recomendación. Se observó una muy baja tasa de adecuación al consumo de frutas y verduras que se traslada a un consumo subóptimo de fibra en gramos / día $(X=9 \mathrm{~g} / \mathrm{d}$, $\mathrm{IDR}=28 \mathrm{~g} / \mathrm{d}$ ).

La Encuesta Nacional de Nutrición y Salud (ENNyS), realizada en el año 2005 en niñas de 10 años de edad, también halló una distribución armónica de macronutrientes. Comunica adicionalmente que el consumo de frutas y verduras en niñas de 10 años de edad, tanto en todo el país como en las diferentes regiones del país analizadas, fue notablemente inferior al recomendado por las Guías Alimentarias para la Población Argentina. ${ }^{14}$

Las ingestas dietéticas de referencia, que se encuentran en permanente evaluación, surgen de estudios realizados en países desarrollados. $\mathrm{Pa}-$ ra nuestro estudio elegimos las IDR para la población estadounidense como referencia porque son las más utilizadas en estudios poblacionales. Esta referencia no distingue entre sexos; a pesar de que, tanto en este estudio como en la mayoría de las publicaciones, suele observarse que la ingesta calórica y de macronutrientes es más alta en los varones.

TABLA 3. Frecuencia de consumo semanal de alimentos de alta densidad calórica para el total de la población ( $n=1673)$

\begin{tabular}{|c|c|c|c|c|}
\hline \multirow{2}{*}{$\begin{array}{l}\text { Alimentos o grupos } \\
\text { de alimentos de alta } \\
\text { densidad calórica }\end{array}$} & \multicolumn{4}{|c|}{ Frecuencia de consumo semanal (\%) } \\
\hline & Nunca & $1 \mathrm{vez} /$ semana & $2-4$ veces/semana & 1 vez/día o más \\
\hline Hamburguesas/Panchos & 47 & 32 & 20 & 1 \\
\hline Rebozados pollo/Pescado & 44 & 29 & 26 & 1 \\
\hline Choripán* & 69 & 26 & 5 & 0 \\
\hline Pizzas/Empanadas & 17 & 45 & 37 & 1 \\
\hline Galletitas dulces & 14 & 17 & 43 & 26 \\
\hline Golosinas (sin chocolate) & 15 & 14 & 38 & 33 \\
\hline Snacks/Productos "copetín" & 44 & 20 & 26 & 10 \\
\hline Gaseosas/Jugos & 3 & 4 & 20 & 73 \\
\hline Chocolate & 25 & 22 & 38 & 15 \\
\hline Tortas/Facturas & 25 & 38 & 29 & 8 \\
\hline Frituras & 18 & 30 & 49 & 3 \\
\hline Manteca/Aderezos grasos & 24 & 18 & 41 & 17 \\
\hline
\end{tabular}

* Sandwich de pan de tipo francés y chorizo (embutido asado de cerdo). 
Otros estudios en niños de este grupo de edad informan un valor de ingesta calórica similar al hallado en esta población. Rolland-Cachera en Francia, en el año 1996, halló una ingesta calórica de $2200 \mathrm{kcal} /$ día en niños de 10 años. ${ }^{15}$ Huang y cols. (EE.UU.) determinaron una ingesta calórica diaria media de $1817( \pm 15) \mathrm{kcal}$; que coincide con la Encuesta de Nutrición de Madrid 2001-2002 (1905 kcal \pm 337$).{ }^{16}$ No hemos hallado publicaciones previas sobre ingesta en niños de este grupo etario en nuestro país; pero sí encontramos una referencia chilena, del año 2010, donde la ingesta calórica media a los 11 años es de $2135 \mathrm{kcal} /$ día, con una distribución muy similar a la de los niños de Buenos Aires. ${ }^{17}$

Las cifras de sobrepeso y obesidad de la población estudiada revelan cuán importante es analizar el valor calórico total de la dieta de la población pediátrica.

El 50\% de la población analizada supera las ingestas dietéticas de referencia de carbohidratos, proteínas y grasas para la edad (Carbohidratos: $\mathrm{IDR}=275 \mathrm{~g} / \mathrm{d}, \mathrm{X}=307 \mathrm{~g} / \mathrm{d}$. Proteínas: $\mathrm{IDR}=75 \mathrm{~g} / \mathrm{d}, X=88 \mathrm{~g} / \mathrm{d}$. Grasas: IDR= 66,6 g/d, $X=84 \mathrm{~g} / \mathrm{d}$ ).

$\mathrm{Al}$ analizar la calidad de la alimentación en la población se observa que los valores diarios de grasas y carbohidratos coinciden con un alto consumo de galletitas dulces, golosinas, gaseosas / jugos y aderezos con altos contenidos de grasa. Particularmente, la frecuencia de consumo de bebidas dulces en la población estudiada es relevante, ya que el $73 \%$ consume gaseosas y jugos concentrados una o más veces al día. En nuestro estudio, este hallazgo no se ha relacionado con el IMC ni con el desplazamiento de otros alimentos. Sin embargo, en la bibliografía internacional, varios estudios relacionan el consumo de bebidas azucaradas con el sobrepeso y la obesidad. ${ }^{18}$

El bajo consumo de frutas, verduras y fibra hallado en este estudio concuerda con la tendencia mundial en este aspecto. Las publicaciones sobre el tema lo asocian al costo, a la dificultad de su preparación, al menor tiempo disponible para la cocina y a la competencia con otros productos industrializados. ${ }^{19} \mathrm{La}$ asociación entre bajo consumo de frutas y verduras y sobrepeso en la infancia ha sido demostrada por varios autores ${ }^{20}$ así como también la relación entre bajo consumo de fibra y enfermedades crónicas no transmisibles. Los resultados hallados en el presente estudio son lo suficientemente alarmantes como para motivar futuras intervenciones al respecto.

El análisis de la relación entre ingesta y salud ha sido explorada en numerosos informes; pero en su mayoría provienen de países cuyo patrón alimentario depende de su propia cultura, como es el caso de EE.UU., donde alimentos como el helado, las hamburguesas, las gaseosas y los snacks salados estarían fuertemente vinculados con el sobrepeso y una alimentación de bajo contenido de nutrientes en la infancia y en la adolescencia. ${ }^{21}$ Sin embargo, en la Argentina, a pesar de la globalización que atraviesa la cultura, parecen ser las galletitas, los panificados con grasa y las bebidas, los alimentos que aportan mayor tenor graso y azúcar a la dieta. ${ }^{22}$ Una reciente publicación local, que incluye información de países de América del Sur, remarca la importancia del ambiente para favorecer una alimentación saludable y la necesidad de realizar estudios locales tendientes a determinar patrones de ingesta, de los cuales surjan intervenciones propias para frenar el camino hacia la obesidad..$^{23}$

En este trabajo no se ha analizado la relación lineal entre ingesta energética y sobrepeso a nivel individual pero resulta interesante observar la alta prevalencia de sobrepeso y obesidad hallada.

En este grupo de edad, los requerimientos de ingesta podrían ser muy variables entre género e inclusive dependientes del grado de desarrollo puberal.

Este estudio no analizó grupos de alimentos, lo cual podría haber sido interesante a la hora de observar la composición de las dietas; tampoco fueron investigados aspectos vinculados con hábitos alimentarios, como el consumo del desayuno.

El instrumento utilizado para medir la frecuencia de consumo de alimentos y bebidas arroja una información limitada, ya que muestra una modalidad general de alimentación pero no permite el análisis de volúmenes $\mathrm{y}$, además, muestra solo la ingesta de los días de la semana. Para evitar el sesgo de tomar días no comparables entre sí, este instrumento conserva la limitación de no comunicar información representativa de los fines de semana.

La metodología para medir ingesta en estudios poblacionales continúa siendo motivo de controversia para la mayor parte de los investigadores, que centran sus trabajos en la evaluación de ingesta en niños. ${ }^{24,25}$ Autores como Rockett y cols., quienes han realizado una exhaustiva evaluación de los métodos existentes para medir ingesta en adolescentes, llegan a la conclusión de que son pocos los estudios validados y reproducibles, por lo que aún se carece de un método ideal (es decir, que sea corto, fácil de administrar, no costo- 
so y preciso). ${ }^{26} \mathrm{Al}$ respecto, Sidossi sostiene que la metodología ideal no existe hasta el momento y que los métodos validados existentes (recordatorio de $24 \mathrm{~h}$ y frecuencia de consumo semanal), acompañados de un programa informático sobre composición de alimentos, son aceptablemente confiables a la hora de analizar la ingesta alimentaria. ${ }^{27}$ Claramente, ésta es un área de interés científico que aún permanece en desarrollo. Nuestro estudio aporta experiencia en la utilización de una metodología validada que permite comparar los resultados obtenidos con los de otras poblaciones argentinas y de otros grupos etarios similares que surjan en el futuro.

La modalidad de armar una muestra con la propia población y realizar una distribución percentilar de la ingesta permitió analizar a la población estudiada no solo en función de la media, sino también mirar sus extremos.

Este es el primer estudio que revela información sobre la ingesta alimentaria de niños de 1012 años del área metropolitana de Buenos Aires. Consideramos que contar con esta información permitirá futuras aplicaciones, tales como:

1. Aportar información para la construcción de ingestas dietéticas de referencia nacionales.

2. Colaborar con el planeamiento de programas de prevención de obesidad y otras enfermedades crónicas no transmisibles.

3. Permitir la implementación de programas de educación nutricional a favor de una alimentación más saludable.

\section{CONCLUSIONES}

Los resultados hallados en esta investigación (una alta prevalencia de sobrepeso y obesidad y una ingesta calórica excesiva en un porcentaje de la población que alcanza el $50 \%$ ) justifican, con información científica, la imperiosa necesidad de trabajar sobre los hábitos alimentarios de los niños y niñas desde edades tempranas de la vida.

\section{Agradecimientos}

A la Dirección General de Escuelas, tanto de la Ciudad Autónoma de Buenos Aires como de la Provincia de Buenos Aires; y, en particular, a los directores y maestros que, al abrir las puertas de sus escuelas y aulas, confiaron en que la investigación en salud tiene como destinatario final a la comunidad. Queremos agradecer también a los niños que, con entusiasmo y paciencia, han colaborado y nos han entregado su valioso tiempo de estudio y juego.

\section{BIBLIOGRAFÍA}

1. Singh AS, Mulder C, Twisk JW, van Merchelen W, et al. Tracking of childhood overweight into adulthood: a systematic review of the literature. Obes Rev 2008;9(5):474-88.

2. Giskes K, Kamphiuis CB, van Lenthe FJ, Kremer SS, et al. A systematic review of associations between environmental factors, energy and fat intakes among adults: is there evidence for environments that encourage obesogenic dietary intakes? Public Health Nutr 2007;10(10):1005-17.

3. Aranceta Bartrina J, Serra-Majem L, Pérez-Rodrigo C, et al. Nutrition risk in the child and adolescent population of the Basque country: the enKid Study. Br J Nutr 2006;96(Suppl 1):S58-66.

4. INDEC, CNPV. Censo Nacional de Población y Vivienda, Ministerio de Economía, República Argentina. 1991.

5. INDEC, CNPHV: Censo Nacional de Población, Hogares y Viviendas, Ministerio de Economía. República Argentina. 2001.

6. Vásquez M, Witriw A. Modelos visuales de alimentos y tablas de relación peso/volumen. Buenos Aires: Universidad de Buenos Aires; 1997.

7. Mazzei M, Pachulu M. Tabla de Composición Química de los Alimentos CENEXA, Buenos Aires: CENEXA (Centro de Endocrinología Experimental Aplicada. UNLP - CONICET) - FEIDEN (Fundación para la promoción de la Educación y la Investigación en Diabetes y Enfermedades de Nutrición); 1995.

8. IOM, I.O.M. Dietary reference intakes for energy, carbohydrate, fiber, fat, fatty acids, cholesterol, protein, and amino acids (macronutrients). F.a.N. Board, Editor. Washington, DC: The National Academies; 2002.

9. IOM, I.O.M. Dietary reference intakes for calcium, phosphorus, magnesium, vitamin D, and uoride. F.a.N. Board. Washington, DC: The National Academies; 1997.

10. IOM, I.O.M. Dietary reference intakes for thiamin, riboavin, niacin, vitaminB6, folate, vitamin B12, pantothenic acid, biotin, and choline. F.a.N. Board. Washington, DC: The National Academies; 2000.

11. IOM, I.O.M. Dietary reference intakes for vitamin A, vitamin $\mathrm{K}$, arsenic, boron, chromium, copper, iodine, iron, manganese, molybdenum, nickel, silicon, vanadium, and zinc. F.a.N. Board, Editor. Washington, DC: The National Academies; 2001.

12. AADyND, et al. Guías alimentarias para la población argentina. Manual de multiplicadores. Buenos Aires, Argentina; 2005.

13. WHO. WHOChild growth standards. 2007. 2007; Disponible en: http://www.who.int/growthref/who2007_bmi_ for_age/en/index.html.

14. Encuesta Nacional deNutricióny Salud (ENNyS). Documento de Resultados. Ministerio de Salud de la Nación; 2007.

15. Rolland-Cachera M, Deheeger M, Bellisle F. Nutritional changes between 1978 and 1995 in 10 year old French children. Int J Obesity 1996;20(4):105-53.

16. Díez-Gañán L, et al. Encuesta de Nutrición Infantil de la Comunidad de Madrid. Consejería de Sanidad de la Comunidad y Madrid; 2008.

17. Liberona Y, Castillo O, Rozowski J. Suficiencia de la dieta y composición corporal en un grupo de niños de 11-14 años de dos clubes deportivos en Santiago de Chile. Rev Chil Nutr 2010;37:145-54.

18. Keller KL, et al. Increased sweetened beverage intake is associated with reduced milk and calcium intake in 3- to 7-year-old children at multi-item laboratory lunches. J Am Diet Assoc 2009;109(3):497-501.

19. Darmon N, Drewnowski A. Does social class predict diet quality? Am J Clin Nutr 2008;87(5):1107-17. 
20. Vergnaud AC, Norat T, Romanguero ND, Mouw T, et al. Fruit and vegetable consumption and prospective weight change in participants of the european prospective investigation into cancer and nutrition-physical activity, nutrition, alcohol, cessation of smoking, eating out of home, and obesity study. Am J Clin Nutr 2012; 95(1):184-93.

21. Sebastian RS, Wilkinson Enns C, Goldman JD. US Adolescents and MyPyramid: associations between fast-food consumption and lower likelihood of meeting recommendations. J Am Diet Assoc 2009;109(2):226.

22. Piaggio $L$, et al. Alimentación infantil en el ámbito escolar: entre patios, aulas y comedores. Salud colectiva 2011;7:199213.
23. Kovalskys IC. Factores obesogénicos vinculados con el ambiente infantil. En: Crecimiento saludable. Entre la desnutrición y la obesidad en el cono sur. Uauy R.C.E, Buenos Aires: Instituto Danone, 2012.

24. Cade J, et al. Development, validation and utilisation of food-frequency questionnaires - a review. Public Health Nutr 2002;5(4):567-87.

25. Wakai K. Areview of food frequency questionnaires developed and validated in Japan. J Epidemiol 2009;19(1):1-11.

26. Rockett HR, Berkey CS, Colditz GA. Evaluation of dietary assessment instruments in adolescents. Curr Opin Clin Nutr Metab Care 2003;6(5):557-62.

27. Sidossis LS. Assessment of energy expenditure and energy intake in children: is it possible? Curr Op Clin Nutr Metab Care 2003; 6(5):499.

\section{FE DE ERRATAS}

En la presentación de casos sobre Lesión renal aguda secundaria a lisis tumoral inducida por esteroides en una adolescente con leucemia linfoblástica aguda. Rol de la alcalinización urinaria y diálisis peritoneal, publicada en Arch Argent Pediatr 2012;110(6):521, por un error involuntario en el resumendebe decir:

\section{Dr. Gustavo Roberto Marína y Dra. Elena Majekb}

\section{RESUMEN}

Una adolescente con leucemia linfoblástica aguda desarrolló un síndrome de lisis tumoral precoz y grave con lesión renal aguda luego de una dosis única y baja de esteroides. La disfunción renal se atribuyó primariamente a una nefropatía por fosfato con nefrocalcinosis debido a elevaciones extremas de este componente en sangre. La alcalinización urinaria probablemente contribuyó a su patogenia. Se utilizó diálisis peritoneal con resolución de la nefrocalcinosis y normalización de la depuración de creatinina.

Palabras clave: lisis tumoral, lesión renal aguda, leucemia, fosfato.

\section{SUMMARY}

An adolescent with acute lymphoblastic leukemia developed an early and severe tumor lysis syndrome with acute kidney injury after a low and single dose of steroids. Renal dysfunction was attributed primarily to phosphate nephropathy with nephrocalcinosis due to extreme elevations of phosphate in blood. Urinary alkalinization probably contributed to this development. We used peritoneal dialysis with resolution of nephrocalcinosis and normalization of creatinine clearance. Key words: Tumor lysis, acute kidney injury, leukemia, phosphate.

http:/ /dx.doi.org/10.5546/aap.2012.e118 